\title{
Measuring parents' readiness to vaccinate themselves and their children against COVID-19
}

Franziska Rees ${ }^{1}$, Mattis Geiger ${ }^{1}$, Lau Lilleholt ${ }^{2}$, Ingo Zettler ${ }^{2}$, Cornelia Betsch ${ }^{3}$, Robert

$$
\begin{gathered}
\text { Böhm }{ }^{2,4} \text {, \& Oliver Wilhelm }{ }^{1} \\
{ }^{1} \text { Ulm University } \\
{ }^{2} \text { University of Copenhagen } \\
{ }^{3} \text { University of Erfurt } \\
{ }^{4} \text { University of Vienna }
\end{gathered}
$$

This manuscript is accepted for publication in Vaccine.

\section{Author's Note:}

Franziska Rees, Institute for Psychology and Education, Ulm University, franziska.rees@uniulm.de; Albert-Einstein-Allee 47, 89081 Ulm, Germany.

Mattis Geiger, Institute for Psychology and Education, Ulm University, mattis.geiger@uniulm.de; Albert-Einstein-Allee 47, 89081 Ulm, Germany.

Lau Lilleholt, Department of Psychology and Copenhagen Center for Social Data Science (SODAS), University of Copenhagen, llj@psy.ku.dk; Øster Farimagsgade 2A, 1353 København K, Denmark.

Ingo Zettler, Department of Psychology and Copenhagen Center for Social Data Science (SODAS), University of Copenhagen, ingo.zettler@psy.ku.dk; Øster Farimagsgade 2A, 1353 København K, Denmark.

Oliver Wilhelm, Institute for Psychology and Education, Ulm University, oliver.wilhelm@uni-ulm.de. Albert-Einstein-Allee 47, 89081 Ulm, Germany.

Cornelia Betsch, Media and Communication Science, University of Erfurt, cornelia.betsch@uni-erfurt.de; Nordhäuser Str. 63, 99089 Erfurt, Germany. Implementation Science, Bernhard Nocht Institute for Tropical Medicine, Hamburg, Bernhard-Nocht-Straße 74, 20359 Hamburg, Germany.

Robert Böhm, Faculty of Psychology, University of Vienna, robert.boehm@univie.ac.at; Universitätsstrasse 7, 1010 Vienna, Austria. Department of Psychology and Copenhagen Center for Social Data Science (SODAS), University of Copenhagen; Øster Farimagsgade 2A, 1353 København K, Denmark.

Correspondence concerning this article should be addressed to Franziska Rees, Institute for Psychology and Education, Ulm University, Albert-Einstein-Allee 47, 89081 Ulm, Germany. Telephone: +49-(0)731/50 31143. Fax: +49-(0)731/50 31149. Email: franziska.rees@uniulm.de 
Supplemental material is publicly available on the Open Science Framework: https://osf.io/6dx8u/ and on the website www.vaccination-readiness.com

Author Contributions: FR, MG, OW, RB, and CB iteratively adapted the 7C scale to child vaccination against COVID-19; LL and IZ commented on the final version. RB, LL, and IZ programmed the study and collected all data. FR analyzed the data together with MG and OW. FR wrote the first draft of the manuscript; MG, OW, RB and CB added sections to the manuscript. All authors reviewed the manuscript, FR drafted the final version.

Acknowledgements: We are grateful to an expert group of the Danish Health Authority for helpful comments on adapting the survey items, and to Cecilie F. Strandsbjerg and Josefine T. Meineche for translations. 


\begin{abstract}
To reach high vaccination rates against COVID-19, children and adolescents should be also vaccinated. To improve childhood vaccination rates and vaccination readiness, parents need to be addressed since they decide about the vaccination of their children. We adapted the 7C of vaccination readiness scale to measure parents' readiness to vaccinate their children and evaluated the scale in a long and a short version in two studies. The study was first evaluated with a sample of $N=244$ parents from the German COVID-19 Snapshot Monitoring (COSMO) and validated with $N=464$ parents from the Danish COSMO. The childhood 7C scale showed acceptable to good psychometric properties in both samples and explained more than $80 \%$ of the variance in vaccination intentions. Additionally, differences in parents' readiness to vaccinate their children against COVID-19 were strongly determined by their readiness to vaccinate themselves, explaining $64 \%$ of the variance. Vaccination readiness and intentions for children changed as a function of the children's age explaining 93\% of differences between parents in their vaccination intentions for their children. Finally, we found differences in correlations of components with self- versus childhood vaccination, as well as between the children's age groups in the prediction of vaccination intentions. Thus, parents need to be targeted in specifically tailored ways, based on the age of their child, to reach high vaccination rates in children. The scale is publicly available in several languages (www.vaccination-readiness.com).
\end{abstract}

Word count: 233/300

Keywords: childhood vaccination, vaccination readiness, COVID-19, vaccine acceptance, parents, questionnaire 


\section{Introduction}

Vaccination is an effective tool for controlling the spread and the impact of infectious diseases [1]. If vaccination rates are sufficiently high, the spread of pathogens can be controlled and limited. To achieve this, people of any age have to be vaccinated. Many efforts focused on understanding adults’ reasons for (not) getting vaccinated [2,3]. Additionally, given that about $30 \%$ of the world's population is younger than 18 years [4] and—in most cases - parents are involved in the decision to vaccinate their child, understanding differences in parents' vaccination decisions is crucial.

Recently, vaccination readiness (i.e., the willingness and readiness getting vaccinated) has received considerable attention. The 5C model of vaccination readiness [5] is grounded in established theoretical frameworks of facilitating and hindering psychological and structural factors of vaccination [e.g., 6], and on health behavior more generally [e.g., 7]. We recently extended this model to seven components (7C) underlying vaccination readiness: Confidence (trust in the safety and effectiveness of vaccines and health authorities), Complacency (seeing a high need for vaccination due to a high perceived risk of the disease), Constraints (not seeing any hurdles in everyday life that hamstring vaccination), Calculation (comparing personal costs against the benefits of receiving a vaccination versus a non-vaccination), Collective responsibility (willingness to protect others by getting vaccinated), and the new factors: Compliance (support for social monitoring and sanctioning of people refusing vaccination), and Conspiracy (a low belief in vaccination-related conspiracy theories and fake news). These seven components have been shown to explain substantial variance in adults' intentions to get vaccinated against the Coronavirus Disease 2019 (COVID-19) [8]. Vaccination status, or the intention to vaccinate oneself, correlates strongly with the intention to vaccinate one's own children $[9,10]$. Yet, little is known about whether the 7C components relate to adults' own vaccination readiness also relate to parents' readiness to vaccinate their children. Regarding components explaining parents' intentions to vaccinate 
themselves and their children, Betsch et al. [5] tested five components of vaccination readiness (confidence, complacency, constraints, calculation, and collective responsibility; see above) and found confidence being the strongest predictor for vaccination uptake against measles-mumps-rubella, human papillomavirus, and influenza. Elsewhere, confidence and constraints were found to underly parents' intentions to get children vaccinated against influenza [11]. Other studies found complacency being an additional important component of vaccination readiness, and the relative importance of all three determinants varied between countries and decisions for oneself versus the child [12,13].

Extending this line of research, we hypothesized that parents' readiness to vaccinate their children against COVID-19 may be predicted by the same seven components that recently have been proposed to relate to adults' vaccination readiness. The relative importance of individual questionnaire items or factors could vary between adults' vaccination readiness for themselves and their children. Events or circumstances, such as the dynamics of the pandemic, the emergence of variants, or the news situation and its perception by individuals could be causes for variation in vaccination readiness. For instance, the willingness to get vaccinated against COVID-19 varied over time for adults [e.g., 14,15] and children [5]. Given differences in vaccination recommendations and access for adults vs. children, this variation was not always uniformly for those groups.

Little is known about how demographic variables relate to components of parental readiness to vaccinate their minor children. Children's age has been shown to relate to parents' vaccination decisions, but the direction of the correlations varies by disease [16-18]. One study found the general population being more skeptic towards COVID-19 child vaccination for younger children [19]. Generally, research found people being more riskaverse when deciding for others than when deciding for themselves [20]. Given the lower risk to suffer from COVID-19 [21] and similar risk for adverse events [22] for children relative to adults, some parents might be more hesitant to vaccinate their children than themselves. 
The European Union initially approved the COVID-19 vaccine for people over 16 in December 2020 [23]. However, the first official recommendation in Germany and Denmark only targeted people over 18 years [24,25]. Approval for children aged 12 to 15 followed in May, 2021 [26]. Vaccination was only recommended for children with preconditions in June and for all children aged 12 to 17 in August [27]. In addition to day-to-day variation in the pandemic course, discrepancies between approval and recommendation might cause variation in vaccination readiness in parents of children in different age groups.

\section{Current research}

The study purpose is fourfold. First, studying parental readiness to vaccinate children requires a psychometrically sound, validated, and efficient measurement tool. We present a tool for assessing childhood vaccination readiness in a long (21 items) and short version (7 items), adapted from the 7C scale of vaccination readiness [8]. We evaluate the measure by using latent variable modeling, suggesting and testing the same factor structure that was shown to account for individual differences in adult vaccination readiness for parental vaccination readiness. Additionally, we test criterion-related validity of the long and short scales by regressing intentions to vaccinate children on the vaccination readiness measurement models.

Second, we examine the level of and relation between parental vaccination readiness for themselves and their children using latent variable modeling. Given that severe COVID19 is more likely for adults than for minors and that people usually tend to be more riskaverse when deciding for others [20], we expect parents' vaccination readiness to be higher for themselves than for their children. Additionally, own vaccination readiness should strongly determine vaccination readiness for one’s children.

Third, we seek to explain variance in parents' childhood vaccination intentions by the parent-children vaccination readiness model and children's age as it is used in approval 
statements of COVID-19 vaccines. We expect vaccination readiness for children to increase stepwise with the given age groups from COVID-19 vaccination approvals.

Last, we exemplify the use of component scores by examining the relative importance of single vaccination readiness components for vaccination intentions for practical use: We calculate bivariate correlations between vaccination intentions and all vaccination readiness components separately for child and adult vaccination.

We addressed these research questions in two studies. In Study 1, we establish the scale psychometrically. In Study 2, we replicate these findings, examine the relationship of parents’ vaccination readiness for themselves and their children, explain vaccination intentions by vaccination readiness and children's age, and examine the relative importance of vaccination readiness components for different age groups.

\section{Study 1: Scale evaluation}

\section{Methods}

\section{Participants and procedure}

Participants were recruited through the German COVID-19 Snapshot Monitoring (COSMO) [28], a repeated cross-sectional survey assessing participants’ perceptions and behaviors related to the COVID-19 pandemic and its associated policies in weekly to biweekly measurements since March 2020. Participants were recruited via a panel provider company. The distribution of age $\times$ sex and residency in German federal states corresponded to those of the German adult population aged between 17 and 74 years. Data collection took place on May 18 and 19, 2021. In total, $N=905$ participants were recruited. Of these, $N=244$ were parents of minors $\left(M_{\text {age }}=38.86, S D=9.72 ; 51.23 \%\right.$ female $)$.

Participants provided information about their demographics, their vaccination readiness for themselves and their oldest child, and the intention to vaccinate their own child(ren). The survey was completed in German. 


\section{Materials}

Demographic characteristics. Participants provided information about their age, sex, and whether they had children (i) below 12 years, (ii) between 12 and 15 years, and (iii) between 16 and 18 years.

COVID-19 childhood vaccination readiness scale. We assessed parents’ childhood vaccination readiness using the 7C scale of vaccination readiness [8] contextualized to COVID-19 and own children. Adaption to COVID-19 and childhood context was done by adding "COVID-19" when referring to the vaccine/vaccination or "child” or "children” to each item (e.g., "I am convinced the appropriate authorities only allow effective and safe (added: COVID-19) vaccines (added: for children).”; 7-point response scale from 1 = strongly disagree to 7 = strongly agree). Parents were instructed to complete the questionnaire while thinking of their oldest child. See the supplement for all items with descriptive statistics. As proposed by Geiger et al. [8], the child-related 7C scale was modeled in a bifactor model [29] with all items loading on a general factor (g-factor) and six orthogonal nested factors for all components besides confidence, which served as reference factor (Figure 1). For the short scale, we selected the same seven items as proposed by Geiger et al. [8]. The short scale was modeled as a g-factor model with correlated residuals for confidence and conspiracy (Figure 2). Both models fit the data acceptably [8].

\section{[FIGURE 1]}

Vaccination intention. Participants were asked about their willingness to vaccinate their children against COVID-19. They were instructed to consider a vaccination against COVID-19 was approved and recommended for their child(ren) by the relevant health authorities and to respond separately for each age group in which they had one or more children. The item reads: "How would you decide, if you had the chance to get these children vaccinated against COVID-19 next week?” (7-point response scale from 1 = definitely not get 
vaccinated to 7 = definitely get vaccinated). If they had children in different age groups, they responded to this item once for each age group.

\section{Analytic approach}

We used confirmatory factor analyses (CFA) to analyze the psychometric properties of the child-related 7C scale. Model fit is deemed acceptable with CFI and $T L I \geq .90, R M S E A \leq$ .08 and $S R M R \leq .11$, and good with $C F I$ and $T L I \geq .95, R M S E A \leq .05$ and $S R M R \leq .08[30-$ 32]. Vaccination intention was predicted by the long scale bifactor model and the short scale g-factor model using structural equation modeling (SEM). Next, children's age was added to regression analyses with dummy-coded age group variables (Table 1). We used robust maximum likelihood (MLR) estimators for CFA and weighted least square mean and variance adjusted (WLSMV) estimators for SEM, because of nonnormal distributions of vaccination intentions. Factors were identified using the effect coding method [33]. All analyses were conducted in R (version 4.03) [34]. Factor analyses were performed with the R package lavaan (version 0.6.8) [35].

\section{[FIGURE 2]}

\section{Results}

\section{Measurement model of the child-related 7C}

Overall, the bifactor model fit the data acceptably: $\chi^{2}(175)=505, p<.001, C F I=$ $.912, T L I=.895, R M S E A=.087, S R M R=.083$. Four residual variances were fixed to 0 to deal with Highwood cases. The factor saturation was large for the g-factor $(\omega=.96)$ andgiven the model architecture-acceptable for nested factors (ranging from $\omega_{\text {Collective responsibility }}$ $=.43$ to $\left.\omega_{\text {Complacency }}=.76\right)$. The short scale model largely fit the data well: $\chi^{2}(13)=35, p=$ $.001, C F I=.974, T L I=.958, R M S E A=.083, S R M R=.038$ and the factor saturation was high $(\omega=.85)$.

\section{Criterion-related validity}


The intention to vaccinate own children was predicted by all factors of the bifactor model $\left(R^{2}=.91\right)$ and the short scale model $\left(R^{2}=.83\right)$. Adding children's age as predictor to the short scale regression analyses had a significant incremental effect $\left(\Delta R^{2}=.10, p<.001\right)$. This was significant for the change to above 12 but not to above 16 years (Table 1 ).

\section{[TABLE 1]}

\section{Discussion}

In Study 1, we evaluated the 7C scale for COVID-19 childhood vaccination by using the same standards as applied for testing the original 7C scale for adults. The scale could be modelled with the same measurement model as the original scale and explained a large amount of variance in intentions to vaccinate own children. Adding children's age increased the amount of explained variance. Please note that some of the loadings (predominantly from the factor calculation) do not adhere to traditional standards. Prior work with the adult version of the 7C scale by Geiger et al. [8] suggests that these loadings fluctuate over the course of the pandemic. We therefore refrained from premature exclusion or major modification of items. Overall, it is remarkable that the model still fits rather acceptably, and that the factors, again, demonstrated strong criterion validity.

\section{Study 2: Parent-children vaccination readiness}

In Study 2 we sought to replicate the results from Study 1 . We examined the relationship between parents' readiness to vaccinate themselves and their own minor children and explained variance in parents' intentions to vaccinate their own children.

\section{Methods}

\section{Participants and procedure}

Participants were recruited from the Danish COSMO branch [36,37]. Participants were invited via the official Danish email system (eboks); invitations were sampled randomly from a larger sample obtained from Statistics Denmark, country-representative for the Danish adult 
population with respect to age and sex. Data collection took place in weeks 22 (31 May to 6 June), 24 (14 to 20 June), 26 (28 June to 4 July), 28 (12 to 18 July), and 30 (26 July to 1 August $)$ of 2021. The complete sample consisted of $N=2,458\left(N_{\text {Sample1 }}=530, N_{\text {Sample } 2}=509\right.$, $\left.N_{\text {Sample3 }}=465, N_{\text {Sample } 4}=457, N_{\text {Sample5 }}=499\right)$ participants. Participants in this study were $N=$ $464\left(N_{\text {Sample } 1}=110, N_{\text {Sample } 2}=98, N_{\text {Sample3 }}=85, N_{\text {Sample4 }}=79, N_{\text {Sample5 }}=92\right)$ with children below 18 years (Table 2).

Participants provided information about their demographics, their own COVID-19 vaccination status, their vaccination intention, as well as demographic information and vaccination intention—-hypothetical in case of approval—for their youngest child. Among other questionnaires not considered here, all participants completed the 7C scale for themselves. Parents also completed the 7C scale for their youngest child. The survey was completed in Danish. The adaptation to the Danish children-7C was done parallel to the adaptation of the German version (Study 1; see Geiger et al. [8] or www.vaccinationreadiness.com for details on translations). The child-related 7C scale is now available in several languages on https://www.vaccination-readiness.com.

\section{[TABLE 2]}

\section{Materials}

Demographic characteristics. Among other demographic variables, we assessed participants’ age, gender, education, and vaccination status. Vaccination status was assessed with one item "Have you been vaccinated with a COVID-19 vaccine?” (3-point response scale with $1=$ Yes, 2 = No, at the moment the vaccine is not available for me because other parts of the population are ahead of me in the line, $3=$ No, I do not wish to get vaccinated with a COVID-19 vaccine). This item was dichotomized for analyses. Parents' of minor children were also asked about their youngest child's age and sex. 
Vaccination readiness scales. We assessed adult vaccination readiness using the 7C scale [8] contextualized to COVID-19. An example item reads "Political decisions about (added: COVID-19) vaccinations are scientifically grounded” (7-point scale from 1 = strongly disagree to 7 = strongly agree). Child-related vaccination readiness was assessed for the youngest child using the child-related 7C scale introduced in Study 1 (with minor changes in wording of eight items to increase the scale’s precision and psychometric properties; for details, see supplement). Table 3 summarizes the 7C and child-related 7C items.

\section{Vaccination intention against COVID-19 for oneself and for one's own children.}

Vaccination intention was assessed using one item each for oneself ("If a vaccine against the novel coronavirus (COVID-19) becomes available, I would get it”) and for one's youngest child (“If a vaccine against COVID-19 becomes available, I would get my child vaccinated.”). The item was answered on a 7-point scale from $1=$ strongly disagree to $7=$ strongly agree . By mistake, vaccination intention for children was not assessed in week 22.

\section{Analytic approach}

Again, vaccination readiness was modeled in a bifactor model with confidence as reference factor (Figure 1) and as short scale in a g-factor model with correlated residuals for confidence and conspiracy (Figure 2). We used the same criteria to evaluate model fit as in Study 1 . We compared parents' vaccination readiness for themselves and their children on a manifest level and predicted child-related vaccination readiness by parents’ vaccination readiness for themselves (parent-child model). Because of the limited sample size, all analyses were conducted with the short scale model, from here on. This parent-child vaccination readiness model and children's age (dummy-coded as in Study 1; Table 1) were used to predict child-related vaccination intentions. 
All analyses were conducted in R (version 4.03) [34]. Factor analyses were performed

with the package lavaan (version 0.6.8) [35]. We used same estimators and methods for factor identification as in study 1.

[TABLE 3]

\section{Results}

\section{Measurement model and criterion validity for COVID-19 childhood vaccination readiness}

For the bifactor model, residual variances for 2 items were fixed to 0 to deal with

Heywood cases [38]. The model (Figure 1) fit the data acceptably: $\chi^{2}(173)=404, p<.001$, $C F I=.952, T L I=.942, R M S E A=.054, S R M R=.046$. The factor saturation was large for the g-factor $(\omega=.94)$ and varied for nested factors ( $\omega_{\text {Constraints }}=.34$ to $\left.\omega_{\text {Compliance }}=.70\right)$. The criterion-related validity was large with $R^{2}=.82$.

The short scale g-factor model (Figure 2) fit the data well to acceptably: $\chi^{2}(13)=50, p$ $<.001, C F I=.960, T L I=.935, R M S E A=.078, S R M R=.031$. The factor saturation $(\omega=.80)$ and criterion-related validity $\left(R^{2}=.82\right)$ were both high.

\section{Parents' vaccination readiness for themselves and for their children}

In general, parents' vaccination readiness was higher for themselves than for their children $\left(M_{\text {Parents }}=4.96,95 \% C I_{\text {Parents }}=[4.86 ; 5.06] ; M_{\text {Children }}=4.08,95 \% C I_{\text {Children }}=[3.92\right.$; 4.25]). This effect was large with $d=.74$. Differences on item and component mean score levels are provided in Table 3.

We predicted COVID-19 childhood vaccination readiness by parental vaccination readiness, using the short scale g-factor and, similar to the bifactor model, residual variances of single items as predictors, using collective responsibility as reference-factor. The model fit the data very well: $\chi^{2}(68)=63, p=.633, C F I=1.000, T L I=.942, R M S E A=.000, S R M R=$ .040. The explained variance in child-related vaccination readiness was high with $R^{2}=.64$. 
When predicting vaccination intentions for own children with the children-7C g-factor

as single predictor, $R^{2}$ was .77 . We added children's age groups using only paths that accounted for variance in vaccination readiness or intentions for children. The model fit the data well (Figure 3): $\chi^{2}(107)=181, p<.001, C F I=.976, T L I=.970, R M S E A=.056$, SRMR $=.063$, and the variance accounted for was large with $R^{2}=.93$. Hence, the incremental validity of adding children's age was large with $\Delta R^{2}=.16(p<.001)$.

\section{Relative importance of vaccination readiness components}

The rank order of bivariate correlations between vaccination readiness components and vaccination intentions differed between parents' vaccination decisions for themselves and their children as well as between children's age groups (Table 4). Calculation was found to be least correlated with vaccination intentions in all groups. In all groups, constraints and collective responsibility belonged to the three components for which the correlation with vaccination intentions was highest. While confidence was more important for children aged below 12, correlations with conspiracy were stronger for children aged 12 years and older.

\section{[FIGURE 3]}

\section{Discussion}

In Study 2, we replicated the findings with regard to the psychometric properties of the child-related 7C scale. We used the 7C short scale to explore the relation of parents' self- and child-related vaccination readiness. In general, parents’ vaccination readiness and vaccination intentions for themselves were higher than for their children. We found parents' vaccination readiness for themselves to account for a large amount of variance in vaccination readiness for their children. This prediction was improved by adding children's age groups. In line with currently valid recommendations by health authorities, parents of the youngest age group had lowest vaccination readiness whereas we found no distinction between the two older age 
groups. Individual differences in vaccination intention could be explained very well by parental vaccination readiness—-for oneself and children—and children's age.

Concerning the relative importance of single vaccination readiness components, we found differences between parents deciding for themselves and their children. Calculation was least important for vaccination intentions regarding oneself and one’s own children. While constraints, collective responsibility, and confidence (in this order) were found to be the most important factors for child vaccination, it was collective responsibility, constraints, and complacency for adult vaccination. This might mirror that COVID-19 vaccination was not approved—and thus, not accessible—for children below 12 years. Also, between age groups the components differed in the rank order of their importance for vaccination intentions. While conspiracy was more important for children older then 12, confidence was more important for children aged below 12. However, it is important to replicate this finding in another sample as this effect might be due to sampling.

\section{[TABLE 4]}

\section{General discussion}

As parents decide whether or not their minor children get vaccinated against COVID19, we need to better understand the individual differences in parental readiness to vaccinate their children. This will allow providing targeted health information to support parental decision making as well as interventions to support vaccine uptake. First, this endeavor requires a sound measurement tool to assess parental readiness to vaccinate their children. Second, we need to test how parents' readiness to vaccinate their children is related to their readiness to vaccinate themselves and, third, how this is affected by other variables. Following those goals, there are three main conclusions from our findings across two studies evaluating a vaccination readiness scale for one's own minor children.

First, both versions showed good psychometric properties and high criterion validity in a German and a Danish Sample. The COVID-19 childhood vaccination readiness scale was 
modelled in the same way as the adults' vaccination readiness scale [8] and we found acceptable to good model fit.

Second, vaccination readiness for children was strongly determined by their parents' readiness to get vaccinated. Investigating the vaccination readiness factor's criterion validity, we found very strong predictive validity of child-related vaccination readiness on the intention to vaccinate their own children when predicting vaccination readiness for children by parents' own vaccination readiness. The vaccination intention item differed between studies but we do not expect this to have implications on the results. Yet, parents' readiness to vaccinate themselves against COVID-19 was higher than their readiness to vaccinate their children with a large effect size. This mirrors current knowledge about COVID-19, suggesting that once infected, children typically show a less severe course of the disease than adults [21], whereas in terms of vaccine adverse events or reactions to the vaccination, these seem limited in both adults and children [22].

Third, we found that child-related vaccination readiness and intentions change as a function of children's age. As expected, the vaccination readiness was lowest for children below 12 years in both samples. The effect of age group increased for children older than 12 and older but not further for children older than 16 years.

\section{Limitations}

Our research is not without limitations. First, since our samples were drawn to maximize representativeness of the respective country populations, and not to oversample parents, the number of parents in both studies was limited. Cell frequencies within the children age-groups over the subsamples were too low to test ordinary multiple group models separately for all subsamples (supplement). Data collection for this study started shortly after COVID-19 vaccination was approved for children aged 12 to 15 years in Europe [26]. Hence, we cannot analyze differences in vaccination readiness from before and after the approval. We did not find differences in vaccination readiness for the two older age groups of children 
but it might be that vaccination readiness increased for children aged 12 to 15 from the time when the vaccine was approved for them. Within the period of data collection for this study, the Danish government started to send vaccination invitation letters to children aged 12 to 15 years [39]. Because of limited sample sizes we could not investigate the effect of these invitations on the vaccination readiness. Similarly, following approval of COVID-19 vaccines, the National Immunization Technical Advisory Group in Germany had issued a recommendation for children aged 12 and older only after collection of the Study 1 data was terminated [27]. Hence, further research should investigate the effect of approval statements and vaccination invitations on vaccination readiness for certain groups. Our study does not allow this. Because we only analyzed several waves of cross-sectional data, we could not test for longitudinal changes. Further research with longitudinal designs is needed and better suited to investigate and understand causes and magnitude of within-person change in vaccination readiness.

Second, factor loadings in the measurement models differed between contexts (children vs. adults) and studies (Study 1 and Study 2). This must be considered carefully as it might indicate problems in the measurement tool. However, as with the adults' version, we do not expect the child-related version to be strictly invariant over the pandemic course. Our knowledge concerning parameter variation over context variables is too limited and we abstain from interpreting minor differences between the children and adult versions as there are many more potential determinants of such differences than pure chance. For instance, these changes might reflect changes in pandemic situations (e.g., current COVID-19 incidence), political or expert recommendations regarding vaccine uptake (especially regarding children in different age groups), the own vaccination status and vaccination experience, personal salience of the pandemic situation, and risk perception for oneself, own children, peers, etc. Another possible cause for volatility in loadings could be non-normal or mixture distributions of qualitatively different subgroups — as highly proactive opponents or 
advocates. On an item and scale level, non-normality was visible in some distributions that were skewed towards high vaccination readiness, supporting these explanations regarding volatile loadings. Nevertheless, it is noteworthy that configural invariance was observed in both contexts and studies over the time. To examine whether the model also holds in groups with extreme opinions will be subject for future research. Further, variation in vaccination readiness might be caused by intercultural differences. The current studies were conducted in Germany and Denmark. We do not expect strict replications or invariance across countries, settings and time, and thus encourage researchers using the 7C scale to derive contextualized predictions relative to some baseline or other results available for the scale.

\section{Implications}

When using the children-7C scale we recommend scoring all items in the direction that high values indicate high vaccination readiness — just like in the adult vaccination readiness scale. Further, we recommend using the short version for survey and panel studies and the long children-7C scale for intervention studies targeting some of the components more strongly than others. In some application contexts, specific aspects of the 7C scale might be pivotal. In this case, we recommend using unit weighted composite mean scores computed from manifest indicators, as they are robust against missing values. However, when investigating effects of interventions on specific components, one must consider the positive manifold among vaccination readiness components. The components of vaccination readiness are highly correlated and can be modelled as a general vaccination readiness factor.

Consequently, any intervention targeting one component should also (to a lower extent) influence other components. Nevertheless, one should consider the relative importance of different components when choosing interventions for adult and childhood vaccination and different children age groups.

In line with other research $[5,8,40]$, confidence and collective responsibility belonged to the most important components determining COVID-19 vaccination intentions. Constraints 
was the most important component in the case of childhood vaccination. This might reflect that the importance of vaccination is prioritized over practical barriers. Parents with children below 12 might not have seen the need to prioritize childhood vaccinations because of lacking recommendations for this age group at the time of our studies. Generally, high perceived barriers to vaccination are often related to lower vaccination intentions [41]. Hence, interventions should facilitate the accessibility and appeal of vaccinations, once recommended. Besides, interventions could also focus on other factors such as confidence and conspiracy trying to increase confidence in vaccines and diminishing false beliefs.

Misinformation about the new mRNA vaccination technology cause special challenges in the way of achieving high vaccination rates against COVID-19 [42]. These safety-related issues seem to be a good starting point for interventions.

Given the strong effect of own vaccination readiness on childhood vaccination readiness and that parents decide about vaccinating their children, parents are an important target group for interventions. Presumably, vaccination decisions are partly determined by their knowledge about vaccines [43-46]. Little is known about the relationship between vaccination readiness, vaccination knowledge, and vaccination decision. Thus, it might be fruitful to explore this relationship and to start tailored interventions about benefits of vaccines for children (and for others they interact with).

Clearly, as adolescents approach adulthood they increasingly influence their vaccination uptake or even make this decision by themselves before adulthood in some countries. Therefore, adolescents might themselves be meaningful target groups for interventions. With an extended instruction giving basic information about vaccines we assume that studying vaccination readiness among children could be feasible with children aged 12 years and older. We consider it worthwhile to investigate whether vaccination readiness among children differs from their parents’ readiness to vaccinate them. If such discrepancies are found, interventions might be adjusted to also focus on children/adolescents. 
404

405

406

407

408

409

410

411

412

413

414

415

416

417

418

419

\section{Conclusions}

To curb the spread of infectious diseases, high vaccine uptake in adults and children is essential. To understand individual differences in parental readiness to vaccinate minor children, we need measurement tools and we need to understand what predicts the readiness to vaccinate children. With the child-related 7C scale, we provide a short and effective tool that is freely available at www.vaccination-readiness.com in several languages. In general, parents' vaccination readiness for themselves is higher than for their children. Parents' readiness to get vaccinated determines their readiness to vaccinate their children and is crucial for actual vaccination behavior. As vaccination readiness also varies by children's age, we should focus on parents with children in critical age groups for specific vaccinations to create effective vaccination interventions.

\section{Conflicts of interest statement}

The authors declare that there are no conflicts of interest. This research was funded by grants from the both the Lundbeck Foundation (R349-2020-592) and the Faculty of Social Sciences, University of Copenhagen to RB and IZ, and the German Research Foundation (BE3970/12-1) to CB. 
421 [1] Thunström L, Ashworth M, Finnoff D, Newbold S. Hesitancy towards a COVID-19

422

423

424 vaccine and prospects for herd immunity. SSRN Electron J 2020. https://doi.org/10.2139/ssrn.3593098.

[2] Betsch C, Böhm R, Chapman GB. Using behavioral insights to increase vaccination policy effectiveness. Policy Insights Behav Brain Sci 2015;2:61-73. https://doi.org/10.1177/2372732215600716.

[3] Larson HJ, de Figueiredo A, Xiahong Z, Schulz WS, Verger P, Johnston IG, et al. The state of vaccine confidence 2016: Global insights through a 67-country survey. EBioMedicine 2016;12:295-301. https://doi.org/10.1016/j.ebiom.2016.08.042.

[4] 2020 World Population Data Sheet 2020.

[5] Betsch C, Schmid P, Heinemeier D, Korn L, Holtmann C, Böhm R. Beyond confidence: Development of a measure assessing the 5C psychological antecedents of vaccination. PLOS ONE 2018;13:e0208601. https://doi.org/10.1371/journal.pone.0208601.

[6] Thomson A, Robinson K, Vallée-Tourangeau G. The 5As: A practical taxonomy for the determinants of vaccine uptake. Vaccine 2016;34:1018-24. https://doi.org/10.1016/j.vaccine.2015.11.065.

[7] Carpenter CJ. A meta-analysis of the effectiveness of health belief model variables in predicting behavior. Health Commun 2010;25:661-9. https://doi.org/10.1080/10410236.2010.521906.

[8] Geiger M, Rees F, Lilleholt L, Santana AP, Zettler I, Wilhelm O, et al. Measuring the 7Cs of vaccination readiness. Eur J Psychol Assess 2021. https://doi.org/10.1027/10155759/a000663.

[9] Brandstetter S, Böhmer MM, Pawellek M, Seelbach-Göbel B, Melter M, Kabesch M, et al. Parents' intention to get vaccinated and to have their child vaccinated against 
COVID-19: cross-sectional analyses using data from the KUNO-Kids health study. Eur J Pediatr 2021. https://doi.org/10.1007/s00431-021-04094-z.

[10] Teasdale CA, Borrell LN, Kimball S, Rinke ML, Rane M, Fleary SA, et al. Plans to vaccinate children for COVID-19: a survey of US parents. Pediatrics; 2021. https://doi.org/10.1101/2021.05.12.21256874.

[11] Zhao M, Liu H, Qu S, He L, Campy KS. Factors associated with parental acceptance of influenza vaccination for their children: the evidence from four cities of China. Hum Vaccines Immunother 2021;17:457-64. https://doi.org/10.1080/21645515.2020.1771988.

[12] Hoogink J, Verelst F, Kessels R, van Hoek AJ, Timen A, Willem L, et al. Preferential differences in vaccination decision-making for oneself or one's child in The Netherlands: a discrete choice experiment. BMC Public Health 2020;20:828. https://doi.org/10.1186/s12889-020-08844-w.

[13] Verelst F, Kessels R, Willem L, Beutels P. No such thing as a free-rider? Understanding drivers of childhood and adult vaccination through a multicountry discrete choice experiment. Vaccines 2021;9:264. https://doi.org/10.3390/vaccines9030264.

[14] Betsch C, Korn L, Felgendreff L, Eitze S, Schmid P, Sprengholz P, et al. Ergebnisse aus dem COVID-19 Snapshot MOnitoring COSMO: Die psychologische Lage. Zusammenfassungen und Empfehlungen Welle 47. Universität Erfurt; 2021.

[15] Szilagyi PG, Thomas K, Shah MD, Vizueta N, Cui Y, Vangala S, et al. National trends in the US public's likelihood of getting a COVID-19 vaccine-April 1 to December 8, 2020. JAMA 2021;325:396. https://doi.org/10.1001/jama.2020.26419.

[16] Dempsey AF, Schaffer S, Singer D, Butchart A, Davis M, Freed GL. Alternative vaccination schedule preferences among parents of young children. PEDIATRICS 2011;128:848-56. https://doi.org/10.1542/peds.2011-0400. 
470 [17] Paek H-J, Shin K-A, Park K. Determinants of caregivers’ vaccination intention with

471

472

473

474

475

476

477

478

479

480

481

482

483

484

485

486

487

488

489

490

491

492

493

494 respect to child age group: a cross-sectional survey in South Korea. BMJ Open 2015;5:e008342. https://doi.org/10.1136/bmjopen-2015-008342.

[18] Zhai Y, Santibanez TA, Kahn KE, Srivastav A, Walker TY, Singleton JA. Rural, urban, and suburban differences in influenza vaccination coverage among children. Vaccine 2020;38:7596-602. https://doi.org/10.1016/j.vaccine.2020.10.030.

[19] Verger P, Peretti-Watel P, Gagneux-Brunon A, Botelho-Nevers E, Sanchez A, Gauna F, et al. Acceptance of childhood and adolescent vaccination against COVID-19 in France: a national cross-sectional study in May 2021. Hum Vaccines Immunother 2021;17:5082-8. https://doi.org/10.1080/21645515.2021.2004838.

[20] Zikmund-Fisher BJ, Sarr B, Fagerlin A, Ubel PA. A matter of perspective: Choosing for others differs from choosing for yourself in making treatment decisions. J Gen Intern Med 2006;21:618-22. https://doi.org/10.1111/j.1525-1497.2006.00410.x.

[21] Zhang J, Dong X, Liu G, Gao Y. Risk and protective factors for COVID-19 morbidity, severity, and mortality. Clin Rev Allergy Immunol 2022. https://doi.org/10.1007/s12016-022-08921-5.

[22] Zou C, Xue X, Qian J. Characteristics and comparison of adverse events of coronavirus disease 2019 vaccines reported to the United States vaccine adverse event reporting system between 14 December 2020 and 8 October 2021. Front Med 2022;9:826327. https://doi.org/10.3389/fmed.2022.826327.

[23] Pinho AC. EMA recommends first COVID-19 vaccine for authorisation in the EU. Eur Med Agency 2020. https://www.ema.europa.eu/en/news/ema-recommends-first-covid19-vaccine-authorisation-eu (accessed July 13, 2021).

[24] Brostrøm S. Information from the Danish Health Authority on vaccination offer against COVID-19 2020. 
495 [25] Vygen-Bonnet S, Koch J, Bogdan C, Harder T, Heininger U, Kling K, et al. Beschluss

496

497

498

499

500

501

502

503

504

505

506

507

508

509

510

511

512

513

514

515

516

517

518 und wissenschaftliche Begründung der ständigen Impfkommission (STIKO) für die COVID-19-Impfempfehlung 2020. https://doi.org/10.25646/7755.2.

[26] Pinho AC. First COVID-19 vaccine approved for children aged 12 to 15 in EU. Eur Med Agency 2021. https://www.ema.europa.eu/en/news/first-covid-19-vaccine-approvedchildren-aged-12-15-eu (accessed May 30, 2021).

[27] Vygen-Bonnet S, Koch J, Armann J, Berner R, Bogdan C, Harder T, et al. Beschluss der STIKO zur 9. Aktualisierung der COVID-19-Impfempfehlung und die dazugehörige wissenschaftliche Begründung 2021. https://doi.org/10.25646/8942.

[28] Betsch C, Wieler L, Bosnjak M, Ramharter M, Stollorz V, Omer S, et al. Germany COVID-19 Snapshot MOnitoring (COSMO Germany): Monitoring knowledge, risk perceptions, preventive behaviours, and public trust in the current coronavirus outbreak in Germany 2020. https://doi.org/10.23668/PSYCHARCHIVES.2776.

[29] Brunner M, Nagy G, Wilhelm O. A tutorial on hierarchically structured constructs: Hierarchically structured constructs. J Pers 2012;80:796-846. https://doi.org/10.1111/j.1467-6494.2011.00749.x.

[30] Bentler PM. Comparative fit indexes in structural models. Psychol Bull 1990;107:23846. https://doi.org/10.1037/0033-2909.107.2.238.

[31] Hu L, Bentler PM. Cutoff criteria for fit indexes in covariance structure analysis: Conventional criteria versus new alternatives. Struct Equ Model Multidiscip J 1999;6:155. https://doi.org/10.1080/10705519909540118.

[32] Steiger JH. Structural model evaluation and modification: An interval estimation approach. Multivar Behav Res 1990;25:173-80. https://doi.org/10.1207/s15327906mbr2502_4. 
[33] Little TD, Slegers DW, Card NA. A non-arbitrary method of identifying and scaling latent variables in SEM and MACS models. Struct Equ Model Multidiscip J 2006;13:59-72. https://doi.org/10.1207/s15328007sem1301_3.

[34] R Core Team. R: A language and environment for statistical computing. Vienna: R Foundation for Statistical Computing; 2020.

[35] Rosseel Y. lavaan : An $R$ package for structural equation modeling. J Stat Softw 2012;48. https://doi.org/10.18637/jss.v048.i02.

[36] Böhm R, Lilleholt L, Zettler I, COSMO Denmark Group. Denmark COVID-19 Snapshot MOnitoring (COSMO Denmark): Monitoring knowledge, risk perceptions, preventive behaviours, and public trust in the current coronavirus outbreak in Denmark 2020. https://doi.org/10.23668/PSYCHARCHIVES.2795.

[37] Zettler I, Lilleholt L, Böhm R, Gondan M. Comparing responses in repeated crosssectional and panel studies: Results across eight weeks during the first COVID-19 lockdown in Denmark. Psychol Assess 2021;33:691. https://doi.org/10.1037/pas0001048.

[38] Kolenikov S, Bollen KA. Testing negative error variances: Is a Heywood case a symptom of misspecification? Sociol Methods Res 2012;41:124-67. https://doi.org/10.1177/0049124112442138.

[39] Brown EA. Covid-19 vaccine: Denmark offers Pfizer jab to children ages 12-15. Local Den 2021.

[40] Burke PF, Masters D, Massey G. Enablers and barriers to COVID-19 vaccine uptake: An international study of perceptions and intentions. Vaccine 2021;39:5116-28. https://doi.org/10.1016/j.vaccine.2021.07.056.

[41] Degarege A, Krupp K, Fennie K, Srinivas V, Li T, Stephens DP, et al. An integrative behavior theory derived model to assess factors affecting HPV vaccine acceptance using 
structural equation modeling. Vaccine 2019;37:945-55.

https://doi.org/10.1016/j.vaccine.2019.01.012.

[42] Löffler P. Review: Vaccine myth-buster - cleaning up with prejudices and dangerous misinformation. Front Immunol 2021;12:663280. https://doi.org/10.3389/fimmu.2021.663280.

[43] Betsch C, Wicker S. E-health use, vaccination knowledge and perception of own risk: Drivers of vaccination uptake in medical students. Vaccine 2012;30:1143-8. https://doi.org/10.1016/j.vaccine.2011.12.021.

[44] Lewis T. Influence of parental knowledge and opinions on 12-month diphtheria, tetanus, and pertussis vaccination rates. Arch Pediatr Adolesc Med 1988;142:283. https://doi.org/10.1001/archpedi.1988.02150030053018.

[45] Pavia M, Rita Foresta M, Carbone V, Angelillo IF. Influenza and pneumococcal immunization in the elderly: knowledge, attitudes, and practices among general practitioners in Italy. Public Health 2003;117:202-7. https://doi.org/10.1016/S00333506(03)00066-0.

[46] Zingg A, Siegrist M. Measuring people’s knowledge about vaccination: Developing a one-dimensional scale. Vaccine 2012;30:3771-7. https://doi.org/10.1016/j.vaccine.2012.03.014. 


\section{Figure 1}

Confirmatory factor analysis for Childhood 7C scale in Study 1 and Study 2.

Study 1: $\chi^{2}(175)=505, p<.001, C F I=.912, T L I=.895, R M S E A=.087, S R M R=.083$

Study 2: $\chi^{2}(173)=404, p<.001, C F I=.952, T L I=.942, R M S E A=.054, S R M R=.046$

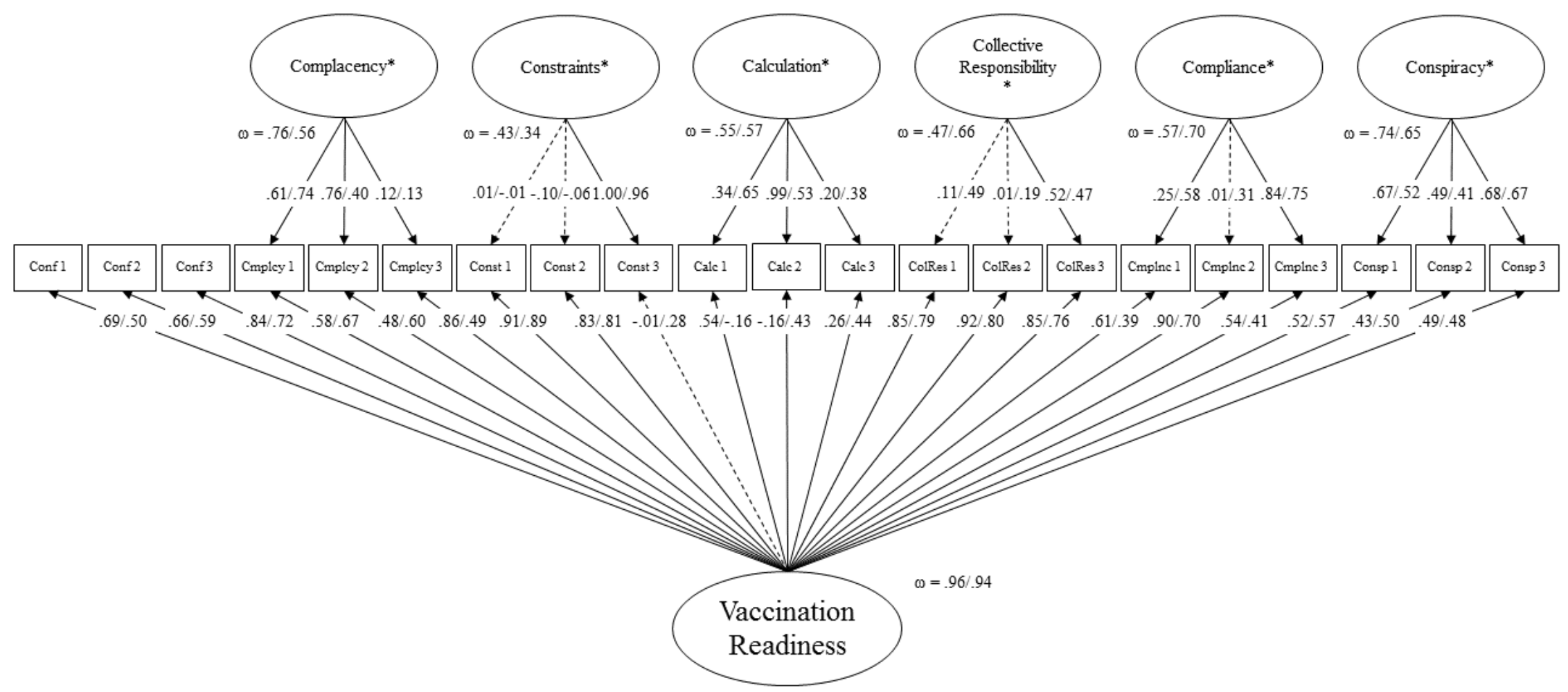

Note. All coefficients are standardized. Loadings not significant on $\alpha=.05$ are depicted as dashed lines. Values belong to Study $1 /$ Study 2 . Conf $=$ Confidence, Cmpcy $=$ Complacency, Const $=$ Constraints, Calc $=$ Calculation, Colr $=$ Collective responsibility, Cmpli $=$ Compliance, Consp $=$ Conspiracy. $N_{S t u d y 1}=244, N_{S t u d y 2}=464$. In Study 1 , for const_03, calc_02, colr_03, and cmpli_03 were fixed to 0 and in Study 2 residual variances for cmpcy_01 and const_03 were fixed to 0. 


\section{Figure 2}

\section{Confirmatory factor analysis for Childhood}

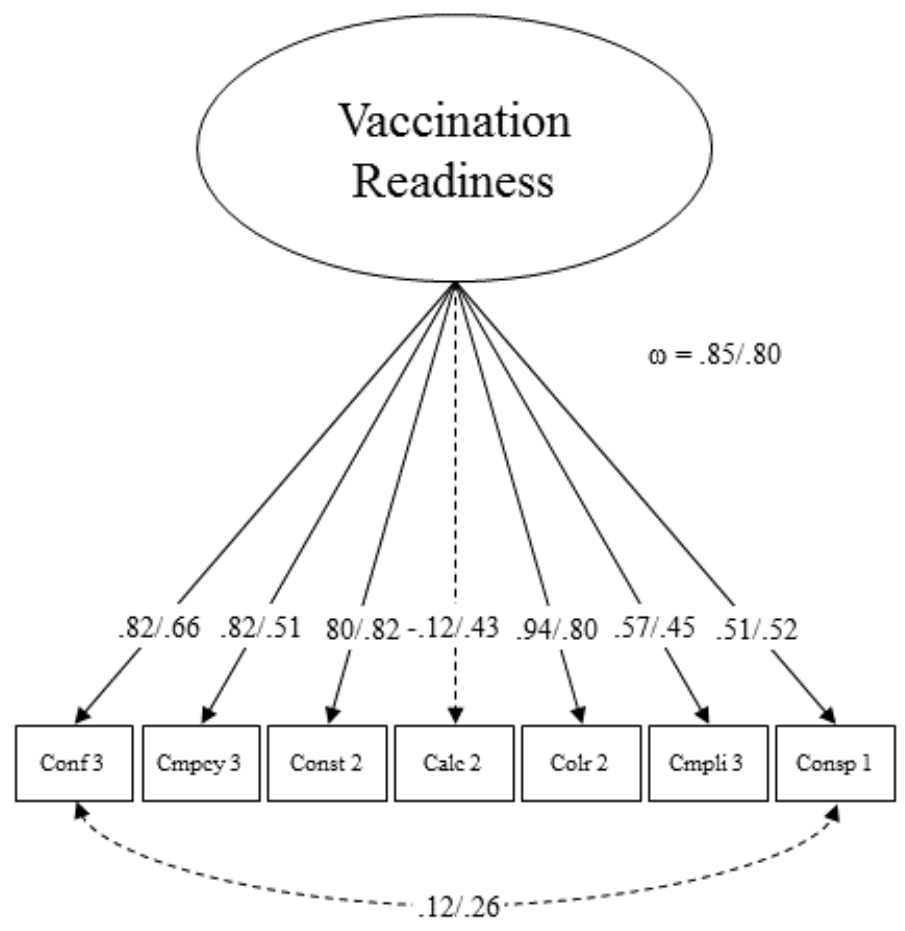

Study 1: $\chi^{2}(13)=35, p=.001, C F I=.974, T L I=.958, R M S E A=.083, S R M R=.038$

Study 2: $\chi^{2}(13)=50, p<.001, C F I=.960, T L I=.935, R M S E A=.078, S R M R=.031$

Note. All coefficients are standardized. Dashed lines indicate values that are not significant in Study 1. Values belong to Study 1/ Study 2. Conf $=$ Confidence, Cmpcy $=$ Complacency, Const $=$ Constraints, Calc $=$ Calculation, Colr $=$ Collective responsibility, Cmpli $=$ Compliance, Consp $=$ Conspiracy. $N_{S t u d y 1}=244, N_{S t u d y 2}=464$. 


\section{Figure 3}

Prediction of intention to vaccinate children with Parent-Child-7C, and children's age in Study 2

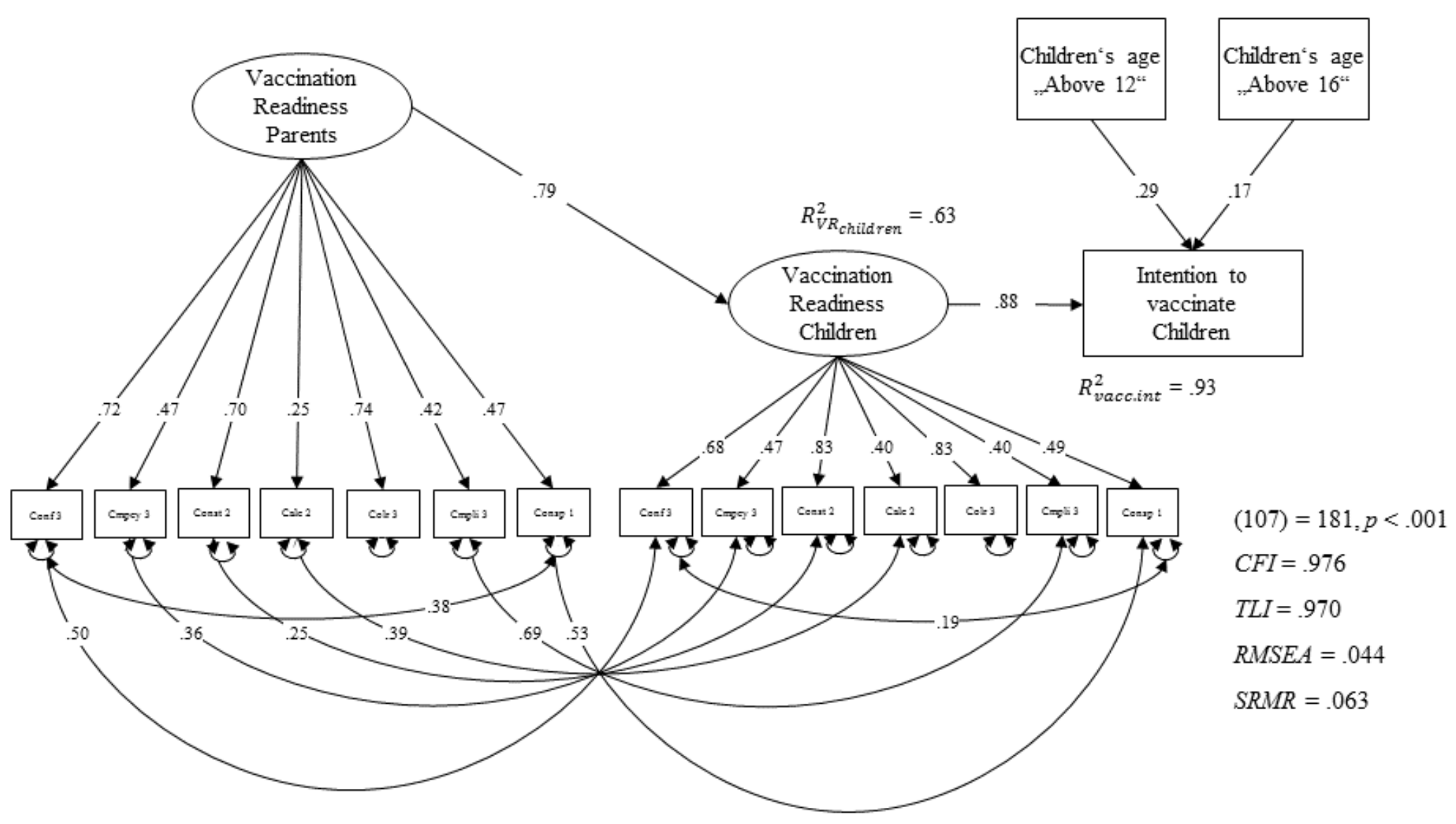

Note. $N=354$, only participants from weeks $24,26,28$, and 30 were included in these analyses as vaccination intention for children was not assessed in week 22. Conf $=$ Confidence, Cmpcy $=$ Complacency, Const $=$ Constraints, Calc $=$ Calculation, Colr $=$ Collective responsibility, Cmpli $=$ Compliance Consp $=$

Conspiracy. Children’s age group was dummy coded with dummy 1: “Above 12” and dummy 2: “Above 16”. 
Table 1

Regression weights for latent regression analyses with the Children-7C short scale in study 1

\begin{tabular}{|c|c|c|c|c|c|c|}
\hline Variable & & Dummy Coding & & $\beta$ & $p$ & $R^{2}$ \\
\hline Model 1 & & & & & & .832 \\
\hline g & & & & .912 & $<.001$ & \\
\hline Model 2 & & & & & & .933 \\
\hline g & & & & .912 & $<.001$ & \\
\hline Children's age group & Below 12 & 12 to 15 & 16 to 18 & .306 & $<.001$ & \\
\hline "Above 12" & 0 & 1 & 1 & & & \\
\hline Children's age group & Below 12 & 12 to 15 & 16 to 18 & .020 & .815 & \\
\hline “Above 16” & 0 & 0 & 1 & & & \\
\hline
\end{tabular}

Note. $N=244, N_{B 12}=144, N_{12-15}=56, N_{16-18}=44$. 
Table 2

Sample characteristics for the complete sample and subsamples in Study 2

\begin{tabular}{|c|c|c|c|c|c|c|c|c|c|c|c|c|}
\hline & \multicolumn{2}{|c|}{ Complete sample } & \multicolumn{2}{|c|}{$\begin{array}{c}\text { Subsample 1: } \\
\text { Week } 22\end{array}$} & \multicolumn{2}{|c|}{$\begin{array}{c}\text { Subsample 2: } \\
\text { Week } 24\end{array}$} & \multicolumn{2}{|c|}{$\begin{array}{c}\text { Subsample 3: } \\
\text { Week } 26\end{array}$} & \multicolumn{2}{|c|}{$\begin{array}{c}\text { Subsample 4: } \\
\text { Week } 28\end{array}$} & \multicolumn{2}{|c|}{$\begin{array}{c}\text { Subsample 5: } \\
\text { Week } 30\end{array}$} \\
\hline & Parents & Children & Parents & Children & Parents & Children & Parents & Children & Parents & Children & Parents & Children \\
\hline$N$ & \multicolumn{2}{|c|}{464} & \multicolumn{2}{|c|}{110} & \multicolumn{2}{|c|}{99} & \multicolumn{2}{|c|}{86} & \multicolumn{2}{|c|}{79} & \multicolumn{2}{|c|}{92} \\
\hline \multicolumn{13}{|l|}{ Gender } \\
\hline Female & 270 & 234 & 60 & 54 & 57 & 48 & 49 & 39 & 48 & 38 & 56 & 55 \\
\hline Male & 194 & 230 & 50 & 56 & 41 & 50 & 36 & 46 & 31 & 41 & 36 & 37 \\
\hline \multicolumn{13}{|c|}{ Vaccination status } \\
\hline Yes & 300 & - & 32 & - & 54 & - & 62 & - & 71 & - & 82 & - \\
\hline No & 164 & - & 78 & - & 45 & - & 23 & - & 8 & - & 10 & - \\
\hline \multicolumn{13}{|c|}{ Vaccination intention } \\
\hline$M$ & 6.36 & 4.68 & 6.23 & - & 6.64 & 4.94 & 6.28 & 4.39 & 6.65 & 4.72 & 6.04 & 4.65 \\
\hline$S D$ & 1.41 & 2.18 & 1.52 & - & 0.98 & 1.99 & 1.53 & 2.15 & 0.86 & 2.33 & 1.82 & 2.27 \\
\hline
\end{tabular}


Table 3

7C items in COVID-19 specific and Children COVID-19 specific versions with descriptive statistics in Study 2

\begin{tabular}{|c|c|c|c|c|c|c|c|c|}
\hline \multirow[b]{2}{*}{$\#$} & \multirow[b]{2}{*}{ 7C COVID-19 } & \multirow[b]{2}{*}{ Children-7C COVID-19 } & \multicolumn{2}{|c|}{ Parents } & \multicolumn{2}{|c|}{ Children } & \multicolumn{2}{|c|}{ Cohen's $d$} \\
\hline & & & $M$ & $S D$ & $M$ & $S D$ & $d$ & $S D$ \\
\hline Confidence & & & 5.013 & 1.304 & 4.675 & 1.353 & 0.254 & 1.329 \\
\hline conf_02 & $\begin{array}{l}\text { Political decisions about COVID-19 } \\
\text { vaccinations are scientifically grounded. }\end{array}$ & $\begin{array}{l}\text { Political decisions about COVID-19 childhood } \\
\text { vaccinations are scientifically grounded. }\end{array}$ & 4.649 & 1.740 & 4.319 & 1.771 & 0.188 & 1.756 \\
\hline conf_03 & $\begin{array}{l}\text { I am convinced the appropriate } \\
\text { authorities only allow effective and } \\
\text { safe COVID-19 vaccines. }\end{array}$ & $\begin{array}{l}\text { I am convinced the appropriate authorities only } \\
\text { allow effective and safe COVID-19 vaccines for } \\
\text { children. }\end{array}$ & 5.621 & 1.601 & 5.313 & 1.749 & 0.184 & 1.677 \\
\hline Complacency & & & 5.695 & 1.354 & 4.685 & 1.502 & 0.706 & 1.430 \\
\hline cmpcy_01 & $\begin{array}{l}\text { I do not need a COVID-19 vaccination } \\
\text { because infectious diseases do not hit me } \\
\text { hard. (R) }\end{array}$ & $\begin{array}{l}\text { My child does not need a COVID-19 vaccination } \\
\text { because infectious diseases do not hit him/her hard. } \\
\text { (R) }\end{array}$ & 6.116 & 1.488 & 5.239 & 1.836 & 0.525 & 1.671 \\
\hline cmpcy_03 & $\begin{array}{l}\text { I get vaccinated because it is too risky } \\
\text { to get infected with COVID-19. }\end{array}$ & $\begin{array}{l}\text { I will get my child vaccinated because it is too } \\
\text { risky for him/her to get infected with COVID-19. }\end{array}$ & 4.912 & 1.966 & 3.483 & 1.979 & 0.724 & 1.973 \\
\hline Constraints & & & 5.764 & 1.235 & 4.955 & 1.399 & 0.613 & 1.319 \\
\hline
\end{tabular}


Table 3 (continued)

\begin{tabular}{|c|c|c|c|c|c|c|c|c|}
\hline \multirow[b]{2}{*}{ \# } & \multirow[b]{2}{*}{ 7C COVID-19 } & \multirow[b]{2}{*}{ Children-7C COVID-19 } & \multicolumn{2}{|c|}{ Parents } & \multicolumn{2}{|c|}{ Children } & \multicolumn{2}{|c|}{ Cohen's $d$} \\
\hline & & & $M$ & $S D$ & $M$ & $S D$ & $d$ & $S D$ \\
\hline const_01 & $\begin{array}{l}\text { I will make sure to get my COVID-19 } \\
\text { vaccination in good time. }\end{array}$ & $\begin{array}{l}\text { I will make sure that my child receives the COVID- } \\
19 \text { vaccination in good time. }\end{array}$ & 5.864 & 1.605 & 4.692 & 1.990 & 0.649 & 1.808 \\
\hline const_02 & $\begin{array}{l}\text { Vaccination against COVID-19 is so } \\
\text { important to me that I prioritize } \\
\text { getting vaccinated over other things. }\end{array}$ & $\begin{array}{l}\text { Vaccination against COVID-19 is so important to } \\
\text { me that I prioritize my child getting vaccinated } \\
\text { over other things. }\end{array}$ & 4.905 & 1.981 & 3.834 & 2.021 & 0.535 & 2.001 \\
\hline Calculation & & & 4.373 & 1.574 & 3.068 & 1.395 & 0.878 & 1.487 \\
\hline calc_01 & $\begin{array}{l}\text { I will get vaccinated against COVID-19 } \\
\text { when I do not see disadvantages for me. } \\
\text { (R) }\end{array}$ & $\begin{array}{l}\text { I will get my child vaccinated against COVID-19 } \\
\text { when I do not see disadvantages for him/her. (R) }\end{array}$ & 3.933 & 2.127 & 2.845 & 1.784 & 0.554 & 1.963 \\
\hline calc_03 & $\begin{array}{l}\text { I carefully consider whether I need a } \\
\text { COVID-19 vaccination. (R) }\end{array}$ & $\begin{array}{l}\text { I carefully consider whether my child needs a } \\
\text { COVID-19 vaccination. (R) }\end{array}$ & 5.063 & 2.139 & 3.399 & 2.108 & 0.783 & 2.124 \\
\hline $\begin{array}{l}\text { Collective } \\
\text { responsibility }\end{array}$ & & & 6.014 & 1.343 & 4.797 & 1.818 & 0.761 & 1.598 \\
\hline
\end{tabular}


Table 3 (continued)

\begin{tabular}{|c|c|c|c|c|c|c|c|c|}
\hline \multirow[b]{2}{*}{$\#$} & \multirow[b]{2}{*}{ 7C COVID-19 } & \multirow[b]{2}{*}{ Children-7C COVID-19 } & \multicolumn{2}{|c|}{ Parents } & \multicolumn{2}{|c|}{ Children } & \multicolumn{2}{|c|}{ Cohen’s $d$} \\
\hline & & & $M$ & $S D$ & $M$ & $\overline{S D}$ & $d$ & $S D$ \\
\hline colr_01 & $\begin{array}{l}\text { I also get vaccinated against COVID-19 } \\
\text { because protecting vulnerable risk } \\
\text { groups is important to me. }\end{array}$ & $\begin{array}{l}\text { I also will get my child vaccinated against COVID- } \\
19 \text { because protecting vulnerable groups is important } \\
\text { to me. }\end{array}$ & 5.841 & 1.614 & 4.750 & 2.026 & 0.595 & 1.832 \\
\hline colr_02 & $\begin{array}{l}\text { I see vaccination as a collective task } \\
\text { against the spread of COVID-19. }\end{array}$ & $\begin{array}{l}\text { I see child vaccinations as a collective task against } \\
\text { the spread of COVID-19. }\end{array}$ & 6.207 & 1.311 & 4.797 & 2.003 & 0.833 & 1.693 \\
\hline colr_03 & $\begin{array}{l}\text { I also get vaccinated against COVID-19 } \\
\text { because I am thereby protecting other } \\
\text { people. }\end{array}$ & $\begin{array}{l}\text { I also will get my child vaccinated against COVID- } \\
19 \text { because thereby other people are protected. }\end{array}$ & 5.996 & 1.532 & 4.845 & 1.953 & 0.656 & 1.755 \\
\hline Compliance & & & 3.943 & 1.658 & 3.184 & 1.608 & 0.464 & 1.633 \\
\hline cmpli_02 & $\begin{array}{l}\text { The health authorities should use all } \\
\text { possible means to achieve high } \\
\text { vaccination rates against COVID-19. }\end{array}$ & $\begin{array}{l}\text { The health authorities should use all possible means } \\
\text { to achieve high vaccination rates against COVID-19 } \\
\text { in children. }\end{array}$ & 4.642 & 1.927 & 3.884 & 1.962 & 0.390 & 1.944 \\
\hline cmpli_03 & $\begin{array}{l}\text { It should be possible to sanction } \\
\text { people who do not follow the } \\
\text { vaccination recommendations by } \\
\text { health authorities }\end{array}$ & $\begin{array}{l}\text { It should be possible to sanction parents who do } \\
\text { not follow the vaccination recommendations by } \\
\text { health authorities. }\end{array}$ & 3.341 & 2.004 & 3.041 & 2.008 & 0.149 & 2.006 \\
\hline
\end{tabular}


Table 3 (continued)

\begin{tabular}{|c|c|c|c|c|c|c|c|c|}
\hline \multirow[b]{2}{*}{$\#$} & \multirow[b]{2}{*}{ 7C COVID-19 } & \multirow[b]{2}{*}{ Children-7C COVID-19 } & \multicolumn{2}{|c|}{ Parents } & \multicolumn{2}{|c|}{ Children } & \multicolumn{2}{|c|}{ Cohen's $d$} \\
\hline & & & $M$ & $S D$ & $M$ & $\overline{S D}$ & $d$ & $S D$ \\
\hline Conspiracy & & & 5.396 & 1.330 & 5.159 & 1.327 & 0.178 & 1.329 \\
\hline consp_01 & $\begin{array}{l}\text { Vaccinations against COVID-19 cause } \\
\text { diseases and allergies that are more } \\
\text { serious than COVID-19. (R) }\end{array}$ & $\begin{array}{l}\text { Vaccinations against COVID-19 cause diseases } \\
\text { and allergies in children that are more serious } \\
\text { than COVID-19. (R) }\end{array}$ & 5.599 & 1.487 & 5.110 & 1.493 & 0.328 & 1.490 \\
\hline consp_02 & $\begin{array}{l}\text { Health authorities knuckle down to the } \\
\text { power and influence of pharmaceutical } \\
\text { companies with respect to COVID-19 } \\
\text { vaccinations. (R) }\end{array}$ & $\begin{array}{l}\text { Health authorities knuckle down to the power and } \\
\text { influence of pharmaceutical companies with respect } \\
\text { to COVID-19 child vaccinations.(R) }\end{array}$ & 5.060 & 1.717 & 5.026 & 1.730 & 0.020 & 1.723 \\
\hline consp_03 & $\begin{array}{l}\text { COVID-19 vaccinations contain } \\
\text { chemicals in toxic doses. (R) }\end{array}$ & $\begin{array}{l}\text { COVID-19 vaccinations contain chemicals in toxic } \\
\text { doses for children. (R) }\end{array}$ & 5.528 & 1.620 & 5.341 & 1.557 & 0.118 & 1.589 \\
\hline
\end{tabular}

\footnotetext{
Note . Conf = confidence, cmply = complacency, const = constraints, calc = calculation, colr = collective responsibility, cmpli = compliance, consp = conspiracy. A 7-point response scale was used from 1 = strongly disagree to 7 = strongly agree. Parents responded to the Children-7C scale thinking of their youngest child. Confidence, collective responsibility, and compliance relate positively with vaccination readiness and complacency, constraints, calculation, and conspiracy relate negatively with vaccination readiness. To avoid confusion, all items should be scored so that high values indicate high vaccination readiness. Items that must be reverse coded are marked with an (R). Items of the short scale are marked bold.
} 
Table 4

Bivariate correlations of manifest Children-7C component scores with vaccination intentions in Study 2

\begin{tabular}{lccccr}
\hline & All children & $<12$ & $12-15$ & $16-18$ & Parents \\
\hline Confidence & 0.617 & 0.626 & 0.588 & 0.449 & 0.525 \\
Complacency & 0.591 & 0.616 & 0.596 & 0.379 & 0.555 \\
Constraints & 0.777 & 0.758 & 0.809 & 0.667 & 0.628 \\
Calculation & 0.330 & 0.288 & 0.373 & 0.115 & 0.357 \\
Collective responsibility & 0.729 & 0.715 & 0.847 & 0.457 & 0.662 \\
Compliance & 0.508 & 0.555 & 0.501 & 0.270 & 0.375 \\
Conspiracy & 0.451 & 0.409 & 0.625 & 0.527 & 0.508 \\
Total vaccination readiness & 0.780 & 0.787 & 0.807 & 0.591 & 0.717 \\
& & & & & \\
\hline
\end{tabular}

Note. $N_{\text {Children }}=354$ since vaccination intentions for children have not been assessed in week 22, $N_{<12}=219, N_{12-15}$ $=81, N_{16-18}=54, N_{\text {Parents }}=464$. All vaccination readiness indicators are mean scores of respective components. 